\title{
Prospects of Semiconductor Terahertz Pulse Sources
}

\author{
Gyula Polónyi, Mátyás I. Mechler, János Hebling, Member, IEEE, and József A. Fülöp
}

\begin{abstract}
Extremely high pump-to-terahertz (THz) conversion efficiencies up to $0.7 \%$ were demonstrated in recent experiments with ZnTe THz pulse sources. Such high efficiencies could be achieved by pumping at an infrared wavelength sufficiently long to suppress both two- and three-photon absorption and the associated free-carrier absorption at $\mathrm{THz}$ frequencies. Here, highfield high-energy THz pulse generation by optical rectification in semiconductor nonlinear materials is investigated by numerical simulations. Basic design aspects of infrared-pumped semiconductor THz sources are discussed. Optimal pumping and phase-matching conditions are given. Multicycle $\mathbf{T H z}$ pulse generation for particle acceleration is discussed.
\end{abstract}

Index Terms - Semiconductor materials, Terahertz materials, Terahertz radiation

\section{INTRODUCTION}

$\mathrm{T}$ ABLE-TOP femtosecond laser sources are now routinely used to generate terahertz $(\mathrm{THz})$ pulses with unprecedented pulse energy and peak electric and magnetic fields. Different parts of the low- $(\sim 0.1-2 \mathrm{THz})$ and mid-frequency $(\sim 2-20 \mathrm{THz})$ bands of the THz range can be accessed by optical rectification (OR) in various nonlinear optical materials. Differencefrequency generation, optical parametric amplification, and $\mathrm{THz}$ radiation from gas plasma generated by two-color laser fields can cover the high-frequency $(\sim 20-200 \mathrm{THz})$ part.

In the low-frequency $\mathrm{THz}$ range $\mathrm{OR}$ in $\mathrm{LiNbO}_{3}(\mathrm{LN})$, using tilted-pulse-front pumping (TPFP) for phase matching [1], has been providing the highest $\mathrm{THz}$ pulse energies [2] and field strengths [3][4] with reported efficiencies up to >3\% [5]. Recently, limitations of this technology have been investigated, which can pose a serious limitation on increasing the $\mathrm{THz}$ yield further. The main reason for these limitations is the large pulsefront tilt angle $\left(\sim 63^{\circ}\right)$ required for phase matching. At medium frequencies OR in organic materials can deliver extremely high field strengths and energies [6].

The potential of semiconductor nonlinear optical materials for high-energy high-field $\mathrm{THz}$ pulse generation by OR has

Date of submission for review.

This work was supported in part by the Hungarian Scientific Research Fund (OTKA) under Grant 113083. J. A. Fülöp acknowledges support from a János Bolyai Research Scholarship (Hungarian Academy of Sciences). The present scientific contribution is dedicated to the 650th anniversary of the foundation of the University of Pécs, Hungary.

Gy. Polonyi is with the MTA-PTE High-Field Terahertz Research Group, Pécs, 7635 Hungary (e-mail: polonyi@ fizika.ttk.pte.hu). been recently recognized [7][8][9][10][11]. Whereas pumping $\mathrm{OR}$ in ZnTe at $0.8 \mu \mathrm{m}$, near its collinear phase-matching wavelength, resulted in maximum $1.5 \mu \mathrm{J} \mathrm{THz}$ pulse energy at $3.1 \times 10^{-5}$ efficiency [12], pumping at $1.7 \mu \mathrm{m}$ wavelength recently resulted in more than two orders of magnitude higher efficiency, as high as $0.7 \%$, and $14 \mu \mathrm{J} \mathrm{THz}$ pulse energy [11]. The reason for the enormous increase in efficiency was the elimination of lower-order $\left(2^{\text {nd }}-\right.$ and $3^{\text {rd }}-$ order $)$ multiphoton pump absorption (MPA) at the longer pump wavelength and the associated free-carrier absorption (FCA) in the THz range.

At such longer infrared pump wavelengths, where in semiconductors usually the optical group velocity is larger than the THz phase velocity, TPFP has to be used for phase matching. An important advantage of semiconductors over LN is the much smaller required pulse-front tilt (PFT) angle. It is typically below $30^{\circ}$, in contrast to $\mathrm{LN}$, where it is about $63^{\circ}$. A smaller tilt angle is advantageous for several reasons. Firstly, it causes a smaller variation of the pump pulse duration within the nonlinear medium and enables a larger effective length for $\mathrm{THz}$ generation. This can help to compensate for the smaller nonlinear coefficient of semiconductors. Secondly, a smaller tilt angle significantly reduces the spatial nonuniformity of the interaction length for $\mathrm{THz}$ generation, and consequently that of the $\mathrm{THz}$ beam. Such a spatial inhomogeneity is a serious drawback in case of LN [13][14]. The potentially much better spatial homogeneity in case of semiconductor sources enables an easier increase of the pumped area and the THz energy. Furthermore, the small tilt angle is also advantageous for the realization of a contact-grating (CG) THz source [8][15][16]. Such a CG source has recently been demonstrated in ZnTe [10], resulting in the generation of $3.9 \mu \mathrm{J} \mathrm{THz}$ pulses with up to $0.3 \%$ efficiency. Importantly, the CG technology enables to practically eliminate spatial nonuniformity of the interaction length, which leads to a straightforward scalability of the $\mathrm{THz}$ energy and excellent $\mathrm{THz}$ beam profile and focusability [10]. CG fabrication technology [10] enables grating sizes on the 5 -cm scale. Based on these advantages, it was anticipated that $\mathrm{THz}$ pulses with $>1 \mathrm{~mJ}$ energy and $>20 \mathrm{MV} / \mathrm{cm}$ electric field strength will be achievable with less than $200 \mathrm{~mJ}$ pump energy

M. I. Mechler is with MTA-PTE High-Field Terahertz Research Group, Pécs, 7635 Hungary (e-mail: mechler@ fizika.ttk.pte.hu).

J. Hebling is with the MTA-PTE High-Field Terahertz Research Group, Pécs, 7635 Hungary and with the Institute of Physics and Szentágothai Research Centre, University of Pécs, 7635 Hungary (e-mail: hebling@ fizika.ttk.pte.hu).

J. A. Fülöp is with the MTA-PTE High-Field Terahertz Research Group, Pécs, 7635 Hungary and with the ELI-ALPS, ELI-Hu Non-profit Ltd., Szeged, 6720 Hungary (e-mail: fulop@ fizika.ttk.pte.hu). 
TABLE I

MATERIAL DATA USED IN THE CALCULATIONS AND/OR THEIR SOURCES

\begin{tabular}{lll}
\hline \hline Quantity & ZnTe & GaP \\
\hline $\begin{array}{l}\text { Effective nonlinear } \\
\text { coefficient for OR, } \\
d_{\text {eff }}[\mathrm{pm} / \mathrm{V}]\end{array}$ & $68.5[22]$ & $24.8[23]$ \\
\hline $\begin{array}{l}\text { Refractive index, } \\
\text { optical range }\end{array}$ & {$[24]$} & {$[25]$} \\
\hline $\begin{array}{l}\text { Dielectric function, } \\
\text { THz range }\end{array}$ & $\begin{array}{l}\text { Data from [26]. } \\
\text { Fitted by formula } \\
\text { from [27] }\end{array}$ & {$[27]$} \\
\hline \hline
\end{tabular}

from a (possibly segmented) CG source [10]. Such pump pulses in the wavelength range of $1.7 \mu \mathrm{m}$ to $2.5 \mu \mathrm{m}$, though not available presently, are definitely feasible in the near future from optical parametric amplifiers or infrared laser technology [17][18]. Efficient monolithic semiconductor THz sources can become a key technology for compact $\mathrm{THz}$ particle accelerators [19][20] and other THz high-field applications [21].

Motivated by the promising development of highly efficient semiconductor THz sources utilizing TPFP [9][10][11], in this paper a numerical study is presented on the choice of the most important device parameters. The aim is to give practical guidelines for the selection of the nonlinear material, the pump wavelength, pulse duration, and intensity, as well as crystal thickness and $\mathrm{THz}$ phase matching frequency. The results are applicable both to conventional TPFP setups containing imaging as well as to novel CG devices.

\section{Theoretical Model AND Material Data}

For most of the simulations, the one-dimensional wave equation with the nonlinear polarization was solved in the spectral domain (Model 1). This model, described in more detail in Ref. [7], takes into account the variation of the pump pulse duration with propagation distance due to material and angular dispersions, the absorption in the $\mathrm{THz}$ range due to phonon resonances and FCA, latter caused by MPA of the pump. For the pump, the projected propagation distance onto the $\mathrm{THz}$ propagation direction was used in order to account for the noncollinear phase matching in a TPFP scheme.

In some cases a more sophisticated model (Model 2) was used. In addition to the effects mentioned above it also took into account nonlinear refraction (Kerr effect) for the pump, leading to self-phase modulation (SPM). Down- and up-conversion of the pump field by the $\mathrm{THz}$ field (cascading effects) were also included [14].

The semiconductor nonlinear materials investigated in detail were $\mathrm{ZnTe}$ and GaP. Material data used in the calculations and/or their sources are summarized in Table I. Other material constants used in the calculations, such as electron effective mass and scattering time, are given in Ref. [7]. The frequency dependence of the refractive indices and linear absorption coefficients of these materials in the $\mathrm{THz}$ range are plotted in Fig. 1, together with those for GaSe [27].

The bandgap of ZnTe is $2.26 \mathrm{eV}$ and that of $\mathrm{GaP}$ is $2.27 \mathrm{eV}$, the latter being an indirect one. These are sufficiently large to avoid both two- and three-photon absorption for pump

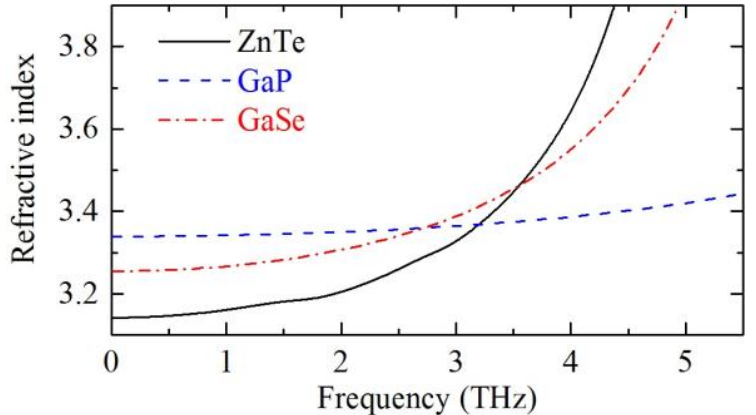

(a)

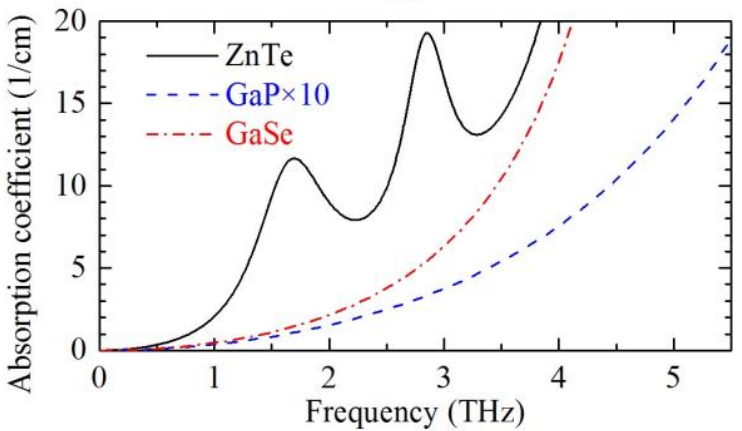

(b)

Fig. 1. Refractive index (a) and linear absorption coefficient (b) of selected semiconductors as functions of the $\mathrm{THz}$ frequency.

wavelengths longer than $1.65 \mu \mathrm{m}$ [11]. GaSe is another suitable material (Fig. 1). It has a slightly smaller bandgap of $2.02 \mathrm{eV}$, requiring a wavelength longer than about $1.85 \mu \mathrm{m}$. These wavelength ranges can be accessed by optical parametric amplifiers driven by Ti:sapphire or Yb lasers, available in many laboratories, or by Ho laser technology operating near $2.05 \mu \mathrm{m}$ [17][18]. Other semiconductors, such as CdTe or GaAs [28], with a significantly smaller bandgap (1.43 eV for both) are less advantageous choices as an even longer pump wavelength would be needed to eliminate both two- and three-photon absorption.

The pump wavelengths considered in the simulations were $1.7 \mu \mathrm{m}$ for $\mathrm{ZnTe}$ and $\mathrm{GaP}$, and $2.05 \mu \mathrm{m}$ for $\mathrm{ZnTe}$. In all cases, the lowest order effective MPA was four-photon absorption (4PA). An estimation for the 4PA coefficient of ZnTe was given in our recent work [11], obtained by fitting simulation results to measured efficiency of $\mathrm{THz}$ generation pumped at $1.7 \mu \mathrm{m}$. Here, we use a slightly different, corrected value of $\beta_{4}=(3 \pm$ 1) $\times 10^{-5} \mathrm{~cm}^{5} / \mathrm{GW}^{3}$, obtained by fitting the same experimental data using Model 1, as shown by the red solid line in Fig. 2. A pump pulse duration of $144 \mathrm{fs}$ and a pump propagation length of $2.9 \mathrm{~mm}$ was used for this calculation. This corresponds to an interaction length for $\mathrm{THz}$ generation of $2.9 \mathrm{~mm} \times \cos (\gamma) \approx 2.5 \mathrm{~mm}$ with a PFT angle of $\gamma \approx 28^{\circ}$ for phase matching at $1 \mathrm{THz}$ in ZnTe. Here, and in the figures below, an average pump intensity at $53 \%$ of the peak intensity is used, given by $I_{\mathrm{p}}=E_{\mathrm{p}} /\left(\tau_{\mathrm{p}} w_{\mathrm{p}}^{2} \pi\right)$, with the pump pulse energy $E_{\mathrm{p}}$, the Fourier-limited pump pulse duration $\tau_{\mathrm{p}}$ (full width at half-maximum), and $1 / e^{2}$ beam radius $w_{\mathrm{p}}$. The saturation of the efficiency, caused by $4 \mathrm{PA}$, with a maximum at about $15 \mathrm{GW} / \mathrm{cm}^{2}$ was observed and well reproduced by the simulation (Fig. 2). At higher increasing pump intensities the 
simulation predicts a more rapidly decreasing efficiency than the observed one. (Because of this deviation between simulation and experiment, measured data corresponding to $>15 \mathrm{GW} / \mathrm{cm}^{2}$ pump intensity were discarded from fitting the value of $\beta_{4}$.)

As a possible reason for this deviation we considered nonlinear refraction (Kerr effect). The nonlinear refractive index of $\mathrm{ZnTe}$ is $n_{2}=1.2 \times 10^{-4} \mathrm{~cm}^{2} / \mathrm{GW}$ [29], which is more than two orders of magnitude larger than that of $\mathrm{LN}$ with a significantly larger bandgap [30]. The comparison of a calculation using Model 1 with 4PA only (red solid line in Fig. 2) and Model 2 with 4PA and pump SPM due to nonlinear refraction (black dashed-dotted line in Fig. 2) reveals a small effect of SPM with a slightly reduced efficiency maximum. Including also cascading effects (blue short-dashed line in Fig. 2) further reduces the efficiency maximum. However, in both cases using Model 2, a similarly rapid drop in efficiency is predicted at high pump intensities than with Model 1, in contrast to the measured slower drop. Varying the value of $\beta_{4}$ in the simulation did not lead to any improvement in this respect. Therefore, we conclude that to predict the location of the maximum $\mathrm{THz}$ generation efficiency it is sufficient to consider 4PA only, i.e. Model 1. In lack of known published data, the same value of $\beta_{4}$ was used for GaP as well. Higherorder MPA was not taken into account due to lack of available absorption coefficient data.

The saturation of 2PA-induced FCA was observed in $\mathrm{ZnTe}$ at high pump fluences [31]. Similarly, saturation of 4PAinduced FCA may also influence $\mathrm{THz}$ generation at higher pump intensities and can be the reason for the observed deviation between experiment and theory. This requires further investigation including the study of ultrafast carrier dynamics, which is beyond the scope of the present work. The pump intensity range most interesting for applications is that up to the maximum of $\mathrm{THz}$ generation efficiency. In this range, the

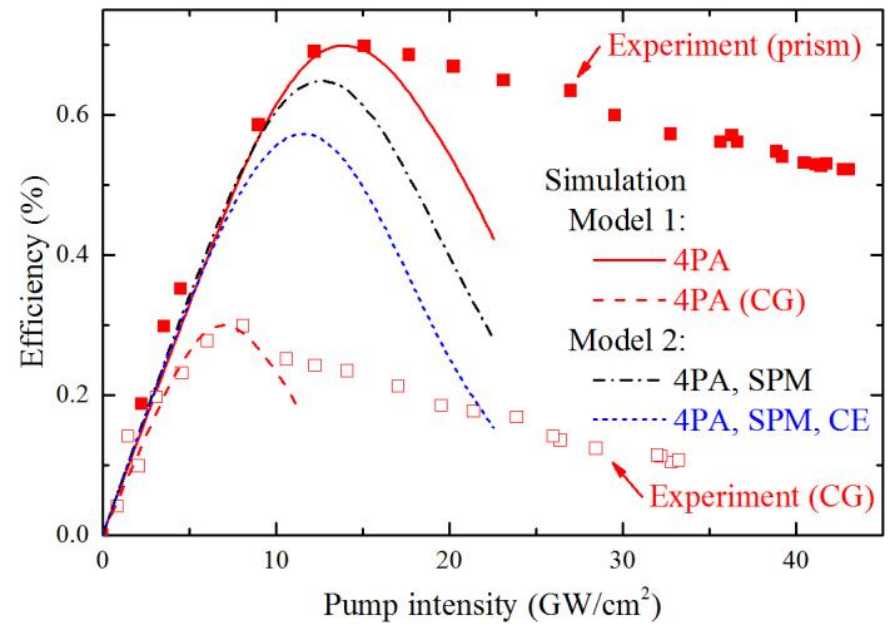

Fig. 2. Measured and calculated THz generation efficiencies as functions of the pump intensity for ZnTe. Full square symbols: measured data with a TPFP setup consisting of a grating, a lens, and a ZnTe prism [11]; empty square symbols: measured data with a ZnTe CG source [10]. The curves show simulation results with Model 1 (red solid line), Model 2 including SPM, but excluding cascading effects (black dashed-dotted line), and Model 2 including both SPM and cascading effects (CE, blue short-dashed line). The red dashed line shows the simulation results for the CG using Model 1 (see text for more details). In all simulations 4PA was included. simple model of FCA [7][32] used here gave a reasonably good agreement with experimental efficiency data (Fig. 2). Furthermore, taking into account the contribution of free carriers to the refractive index (in addition to FCA) had only a little effect on the peak electric field and efficiency, and a moderate effect on the details of the $\mathrm{THz}$ waveform and spectrum. Its effect has been neglected here as it did not influence the general trends emphasized in this work.

We note that the same value of $\beta_{4}$ can be used to fit (Fig. 2, red dashed line) the results of another measurement (Fig. 2, empty square symbols), carried out with a ZnTe CG source [10]. The observed dependence of the $\mathrm{THz}$ generation efficiency is very similar to the previous case [11]. However, the maximum efficiency is located at about half of the pump intensity for the efficiency maximum in the previous case, i.e. at about $8 \mathrm{GW} / \mathrm{cm}^{2}$ rather than about $15 \mathrm{GW} / \mathrm{cm}^{2}$. The reason is the interference of the two diffraction orders \pm 1 from the $\mathrm{CG}$, which doubles the maximum pump intensity. The smaller efficiency is mainly caused by the smaller crystal length for the CG.

\section{RESULTS AND DISCUSSION}

In case of LN theoretical [33] and experimental [34] studies demonstrated the importance of the choice of pump pulse duration for the efficient generation of intense $\mathrm{THz}$ pulses. For the design and future development of intense semiconductor $\mathrm{THz}$ sources the choice of the pump pulse duration is equally important. Below we present a study of effect of pump pulse duration, pump intensity, and crystal length on the achievable $\mathrm{THz}$ peak electric field and waveform, as well as $\mathrm{THz}$ generation efficiency and $\mathrm{THz}$ spectrum. Model 1 is used for the simulations. Results are presented separately for the investigated materials.

\section{A. Zinc Telluride (ZnTe)}

Fig. 3a shows the calculated peak electric field as function of the Fourier-limited pump pulse duration for different pump intensities. Note that the variation of the pulse duration at a constant intensity implies the variation of the pump pulse energy. The pump wavelength was $1.7 \mu \mathrm{m}$. The interaction length was $2.5 \mathrm{~mm}$. In each case, the phase-matching $\mathrm{THz}$ frequency was iteratively fitted to the frequency of the maximum spectral intensity.

At the lowest considered pump intensity $\left(7.5 \mathrm{GW} / \mathrm{cm}^{2}\right)$ the achievable peak electric field first increases with increasing pump pulse duration. A maximum of the peak field is reached for $\sim 325$ fs. For longer pulses the peak field strength decreases. The THz generation efficiency, shown in Fig. 3d as function of the pulse duration, exhibits similar behavior, but the maximum is shifted to a shorter (200 fs) pulse duration. The different optimum pulse durations for field and efficiency can be attributed to the influence of material dispersion in the $\mathrm{THz}$ range. The reason for the initial increase is the more efficient generation of low-frequency $\mathrm{THz}$ spectral components when the pulse duration gets longer (and its spectrum gets narrower). 


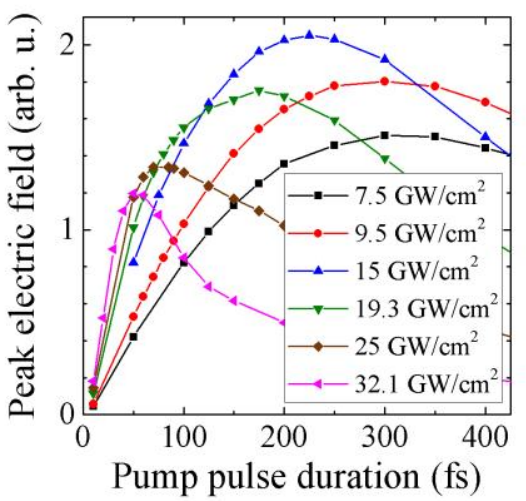

(a)

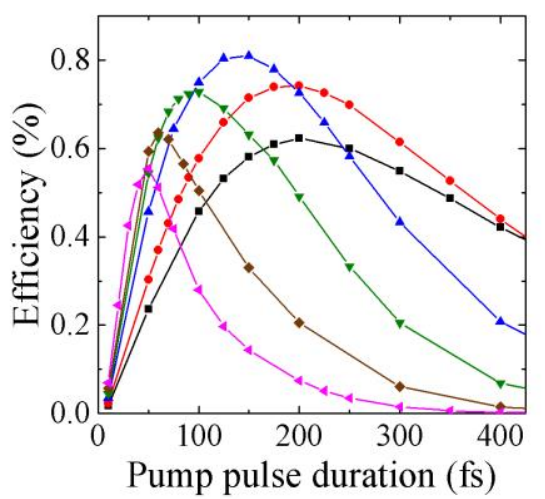

(d)

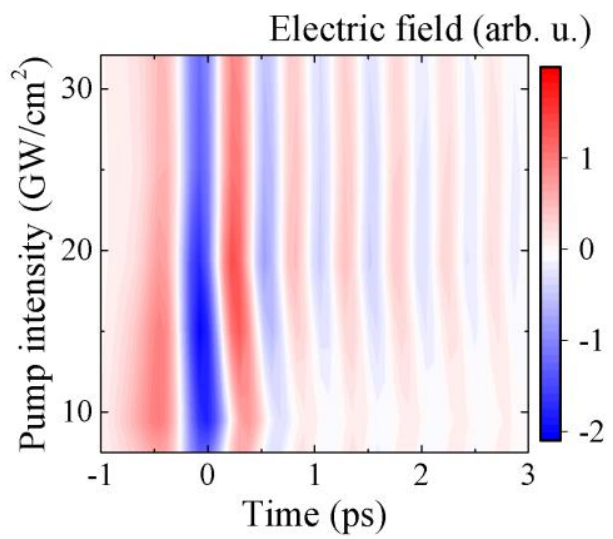

(b)

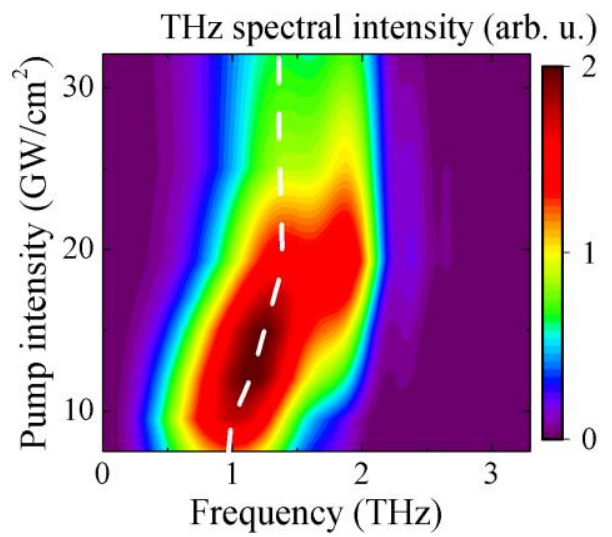

(e)

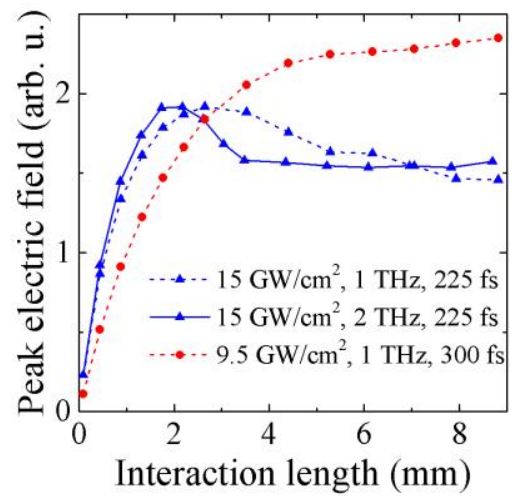

(c)

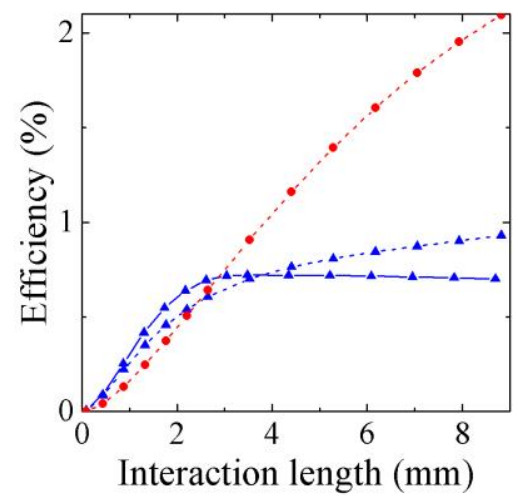

(f)

Fig. 3. Simulation results for ZnTe with $2.5 \mathrm{~mm}$ interaction length, pumped at $1.7 \mu \mathrm{m}$ wavelength. Peak electric field (a) and THz generation efficiency (d) as functions of the Fourier-limited pump pulse duration for different values of the pump intensity. Time dependence of the electric field (b) and spectral intensity (e) of the THz pulses for different pump intensities. The white dashed curve in panel (e) indicates the phase-matching frequency. The THz peak electric field (c) and the efficiency (f) as functions of the interaction length at the indicated pump intensities, pump pulse durations, and phase-matching THz frequencies. Legends for panels (d) and (f) are shown in (a) and (c), respectively.

This is advantageous as the linear absorption coefficient of $\mathrm{ZnTe}$ is smaller at lower frequencies (Fig. 1b). However, the efficiency of OR scales with the square of the $\mathrm{THz}$ frequency [35], counteracting the smaller absorption at lower frequencies. The interplay of these two effects results in the optimum pulse duration.

When the intensity is increased to about $10 \mathrm{GW} / \mathrm{cm}^{2}$, the field and efficiency maxima increase, but the corresponding optimal pulse durations remain unchanged. However, increasing the intensity to $15 \mathrm{GW} / \mathrm{cm}^{2}$ results in the shortening of the optimal pulse durations to 225 fs for the electric field and $150 \mathrm{fs}$ for the efficiency. This indicates the onset of 4PAinduced FCA. The trend of shortening optimal pulse durations, which continues to still higher pump intensities, can be explained by the characteristic spectral dependence of FCA. FCA is strongest at low $\mathrm{THz}$ frequencies and rapidly decreases towards higher frequencies, thereby favoring shorter pump pulses, which can more efficiently generate higher $\mathrm{THz}$ frequencies.

Fig. $3 \mathrm{~b}$ shows the $\mathrm{THz}$ waveform (i.e. the time dependence of the electric field) as function of the pump intensity, corresponding to the respective optimal pulse durations for the highest electric field. The waveform gets chirped due to material dispersion in the $\mathrm{THz}$ range, which is clearly visible especially at higher intensities with higher average $\mathrm{THz}$ frequency. Fig. 3e shows the $\mathrm{THz}$ spectra as function of the pump intensity, corresponding to the respective optimal pulse durations for the highest electric field. The shift of the $\mathrm{THz}$ spectrum to higher frequencies with increasing pump intensity, caused by stronger FCA at low frequencies as mentioned above, can be clearly seen. Frequency components above $\sim 2.5 \mathrm{THz}$ are strongly suppressed by the large linear absorption coefficient of ZnTe (see also Fig. 1b).

As shown in Figs. $3 \mathrm{a}$ and $3 \mathrm{~d}$, the all-over highest electric field and the highest efficiency $(0.81 \%)$ are both achieved at $15 \mathrm{GW} / \mathrm{cm}^{2}$ pump intensity (for $225 \mathrm{fs}$ and $150 \mathrm{fs}$ pulse durations, respectively, as mentioned above). Note that the maximum efficiency was measured at about the same pump intensity using $\sim 150$-fs pulses, as seen in Fig. 2. At higher intensities FCA suppresses $\mathrm{THz}$ generation and the field and efficiency maxima are reduced (Figs. 3a and 3d).

The scaling of the peak electric field and the efficiency with the interaction length is shown in Figs. 3c and 3f. For $15 \mathrm{GW} / \mathrm{cm}^{2}$ pump intensity and $225 \mathrm{fs}$ pulse duration (corresponding to the highest peak electric field in Fig. 3a), the peak field rapidly increases up to almost $3 \mathrm{~mm}(2 \mathrm{~mm})$ 
interaction length for $1 \mathrm{THz}(2 \mathrm{THz})$ phase-matching frequency (Fig. 3c). This is followed by a slower decrease, indicating predominantly the limiting effect of material dispersion. The corresponding efficiency rapidly increases up to about $3.5 \mathrm{~mm}$ $(2.5 \mathrm{~mm})$. Beyond this length, 4PA sets on, prohibiting further substantial increase. The onset of 4PA can be suppressed and a much larger interaction length can be efficiently utilized by reducing the pump intensity to about $9.5 \mathrm{GW} / \mathrm{cm}^{2}$ and using a corresponding longer pulse duration of about $300 \mathrm{fs}$ (cf. Fig. 3a). In this case a higher peak electric field and a steadily increasing efficiency can be achieved even up to $9 \mathrm{~mm}$ length (Figs. 3c and 3f).

Our calculations for $2.05 \mu \mathrm{m}$ pump wavelength show a very similar behavior of the peak electric field strength and efficiency. For example, the latter has a maximum of $0.76 \%$ at $15 \mathrm{GW} / \mathrm{cm}^{2}$ pump intensity and $150 \mathrm{fs}$ pulse duration, similarly to the case of $1.7 \mu \mathrm{m}$. The similar behavior is also retained for a larger crystal length of $5 \mathrm{~mm}$. For $1.7-\mu \mathrm{m}$ pump wavelength, the all-over highest electric field and the highest efficiency $(\sim 1.3 \%)$ are both achieved at a somewhat lower intensity of $12 \mathrm{GW} / \mathrm{cm}^{2}$ for 275 fs and 175 fs pulse durations, respectively. The maximum of the field is about $10 \%$ higher than for the 2.9-mm crystal. The $\mathrm{THz}$ spectra get slightly narrower and lower in center frequency.

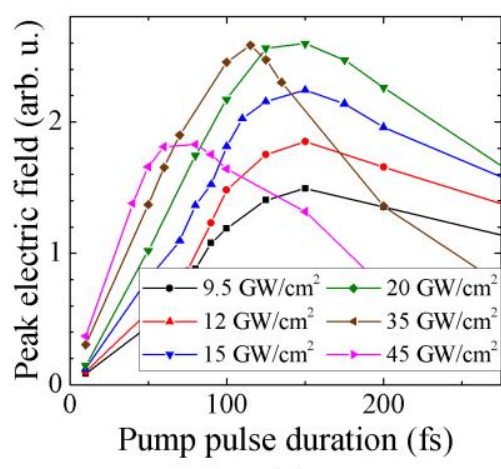

(a)

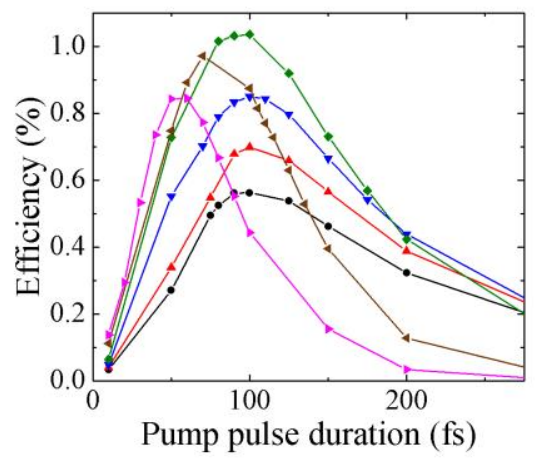

(d)

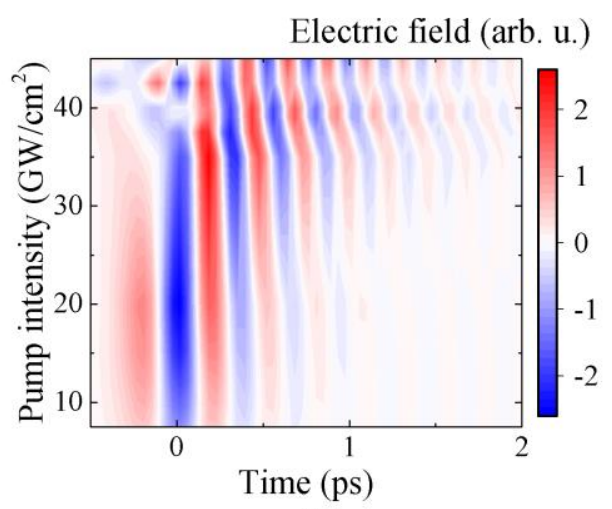

(b)

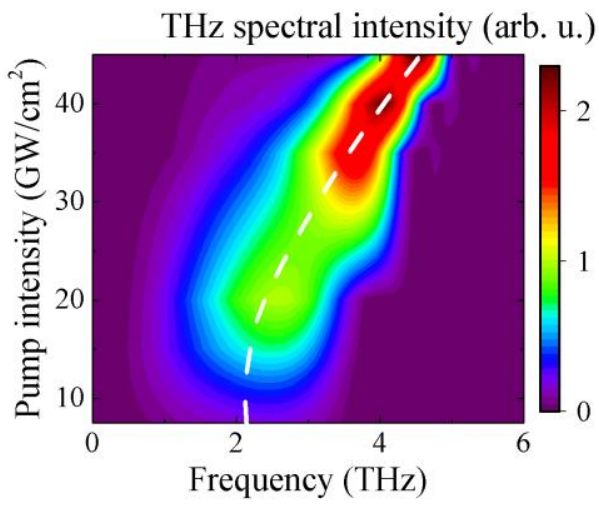

(e)

\section{B. Gallium Phosphide (GaP)}

$\mathrm{GaP}$ has a significantly smaller absorption and dispersion in the $\mathrm{THz}$ range as $\mathrm{ZnTe}$ (Fig. 1). It can also be manufactured in larger sizes and better quality. Its smaller nonlinear coefficient, as compared to that of ZnTe, may be partially compensated by the larger effective interaction length for OR, enabled by the smaller PFT angle and dispersion. Owing to its higher phonon frequency and small linear absorption coefficient, it can be especially suitable for generating higher frequencies in the range of $2-5 \mathrm{THz}$ or even beyond. For these reasons, $\mathrm{GaP}$ is another promising material for intense $\mathrm{THz}$ pulse generation.

Fig. 4a shows the calculated peak electric field as function of the Fourier-limited pump pulse duration for different pump intensities. The pump wavelength was $1.7 \mu \mathrm{m}$ and the interaction length for $\mathrm{THz}$ generation was $2.7 \mathrm{~mm}$. At small pump intensities, the optimal pump pulse duration giving the highest peak fields is about $150 \mathrm{fs}$. This is significantly shorter than in case of ZnTe ( $\sim 325 \mathrm{fs})$. The reason is that the smaller linear absorption and dispersion enable the generation of a significantly broader spectrum up to about $4 \mathrm{THz}$. This is clearly shown by the spectra in Fig. 4e. Higher frequencies can be more efficiently generated by shorter pulses.

There is no change in the optimal pulse duration up to an intensity as high as $20 \mathrm{GW} / \mathrm{cm}^{2}$. The optimum pulse duration for the efficiency in this intensity range is as short as $100 \mathrm{fs}$ (Fig. 4d).

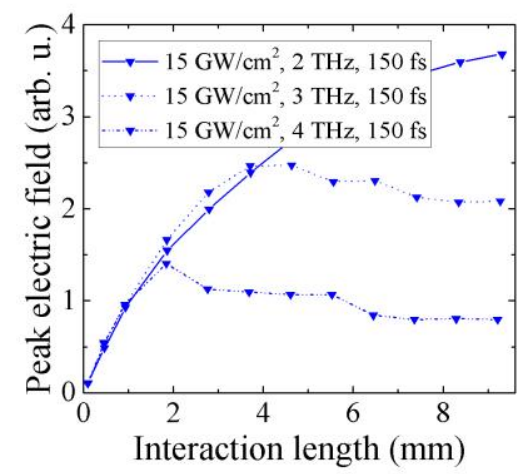

(c)

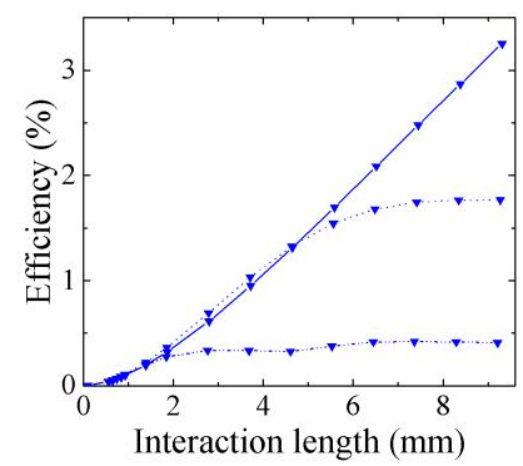

(f)

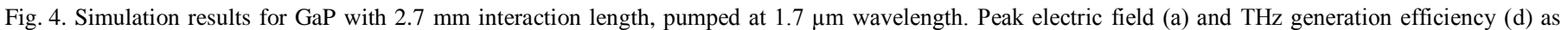

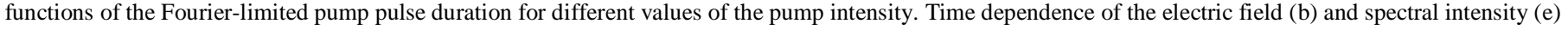

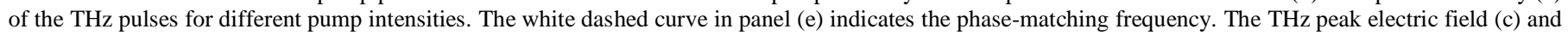

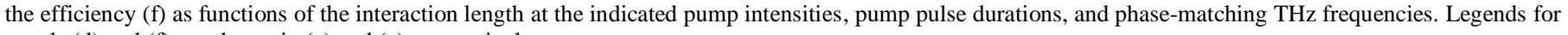
panels (d) and (f) are shown in (a) and (c), respectively. 
The all-over field and efficiency maxima are both reached at about $20 \mathrm{GW} / \mathrm{cm}^{2}$ with $150 \mathrm{fs}$ and $100 \mathrm{fs}$ pulse durations, respectively. This intensity is higher than the corresponding value in $\mathrm{ZnTe}\left(15 \mathrm{GW} / \mathrm{cm}^{2}\right)$. The reason is that the FCA coefficient is smaller in GaP than in ZnTe. Even if the assumed 4PA coefficients are the same, other material parameters (for example the electron effective mass) influencing FCA are different [7].

At still higher intensities the maxima get reduced due to the onset of 4PA. FCA shifts the $\mathrm{THz}$ spectrum to higher frequencies (Fig. 4e) and the optimal pump pulse durations get shorter. The generated higher-frequency $\mathrm{THz}$ pulse gets more susceptible for material dispersion, as shown by the waveforms in Fig. 4b.

Figs. $4 \mathrm{c}$ and $4 \mathrm{f}$ show the dependence of the peak electric field and the efficiency, respectively, on the interaction length for the fixed phase-matching frequencies of $2 \mathrm{THz}, 3 \mathrm{THz}$, and $4 \mathrm{THz}$. Fig. 5 shows the corresponding dependence of the waveforms (a-c) and normalized spectra (d-f) of the $\mathrm{THz}$ pulses on the interaction length. The pump wavelength was $1.7 \mu \mathrm{m}$, the intensity $15 \mathrm{GW} / \mathrm{cm}^{2}$, and the pulse duration $150 \mathrm{fs}$, latter corresponding to the optimum value for the highest peak field, as shown in Fig. 4a.

For phase matching at $2 \mathrm{THz}$, the peak field strength increases monotonically over the whole range of interaction lengths (Fig. 4c), whereas the waveform exhibits a moderate lengthening and chirp (Fig. 5a), owing to the small material dispersion of GaP. Similarly, the efficiency also increases monotonically, above $4 \mathrm{~mm}$ nearly linearly, with the interaction length (Fig. 4f). A very broad spectrum extending beyond $5 \mathrm{THz}$ can be generated in a 1-mm length (Fig. 5d). This spectrum gradually narrows down with increasing length to a bandwidth of less than $3 \mathrm{THz}$ at around $9 \mathrm{~mm}$.

For phase matching at $3 \mathrm{THz}$, the peak field strength increases up to about $4 \mathrm{~mm}$ interaction length, followed by a slow decrease at larger lengths (Fig. 4c). The efficiency increases up to about $6 \mathrm{~mm}$, followed by a range with nearly constant value (Fig. 4f). The effect of material dispersion in the $\mathrm{THz}$ range becomes more pronounced with increasing phasematching frequency and interaction length. On the one hand, dispersion leads to a more strongly chirped waveform, clearly observed in Fig. 5b. On the other hand, it gradually decreases the phase-matching bandwidth (Fig. 5e). Beyond about $6 \mathrm{~mm}$ length the spectrum contracts to a narrow band around the $3 \mathrm{THz}$ phase-matching frequency. This results in a multi-cycle waveform (Fig. 5b).

This trend gets even more pronounced at a still higher phasematching frequency of $4 \mathrm{THz}$. The highest peak field strength is reached at a small interaction length of only $2 \mathrm{~mm}$ (Fig. 4c). Also in efficiency, no substantial increase can be observed beyond this length (Fig. 4f). This is accompanied by a rapid collapse of the initially broad spectrum to a very narrow band (Fig. 5f). The corresponding waveform evolves into a manycycle pulse (Fig. 5c).

These results clearly show the importance of the proper choice of the phase-matching frequency and the crystal length

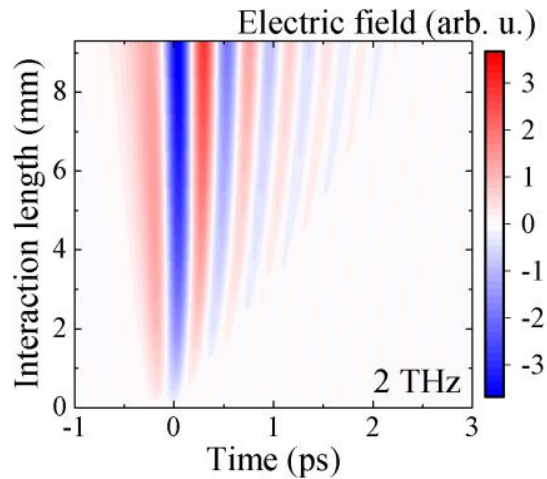

(a)

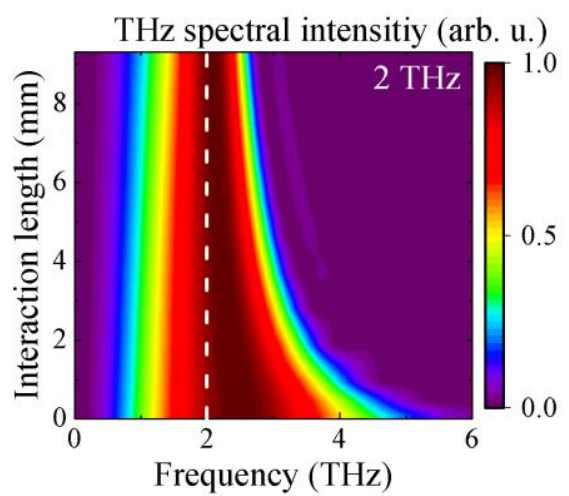

(d)

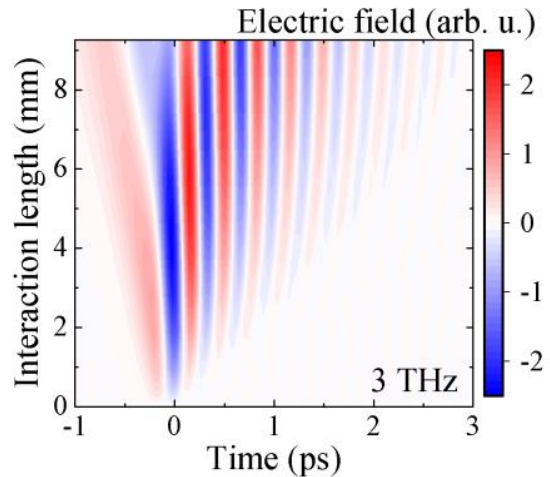

(b)

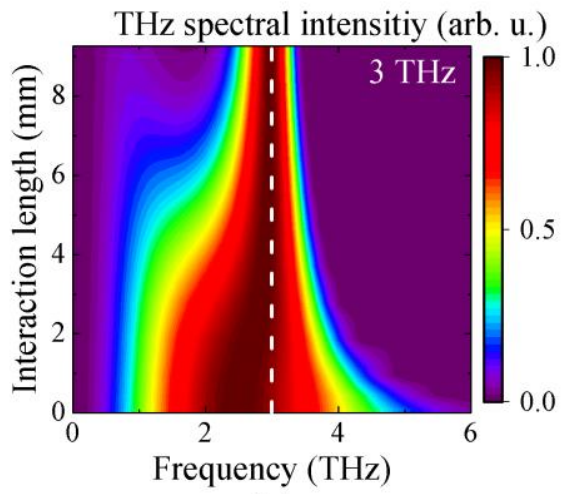

(e)

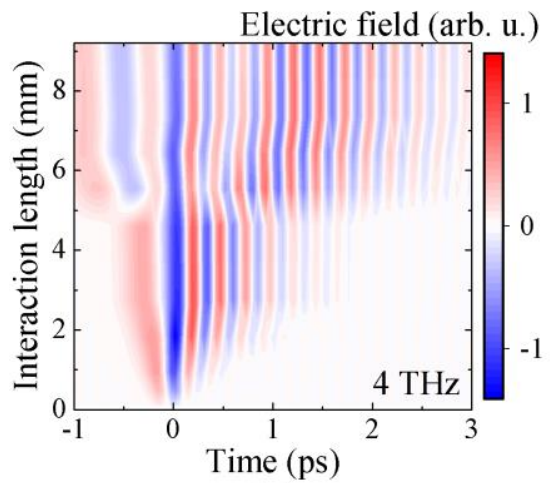

(c)

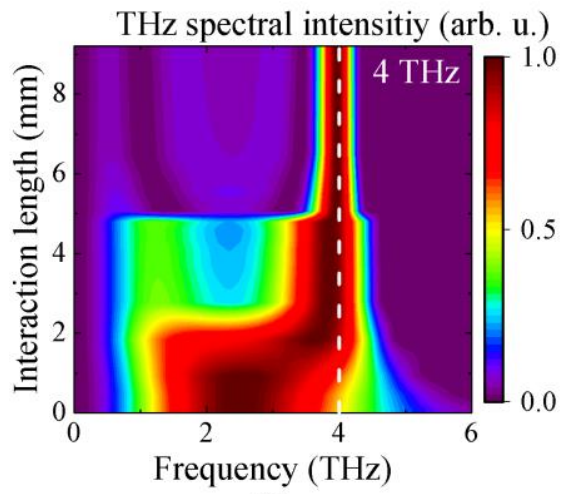

(f)

Fig. 5. Waveforms of THz pulses for GaP with varying thickness at different phase-matching frequencies of $2 \mathrm{THz}$ (a), $3 \mathrm{THz}$ (b), and $4 \mathrm{THz}$ (c). The pump intensity was $15 \mathrm{GW} / \mathrm{cm}^{2}$, the pump wavelength $1.7 \mu \mathrm{m}$, and the pulse duration $150 \mathrm{fs}$. (d)-(f) show the corresponding normalized THz spectra. The white dashed lines indicate the phase-matching frequencies. 


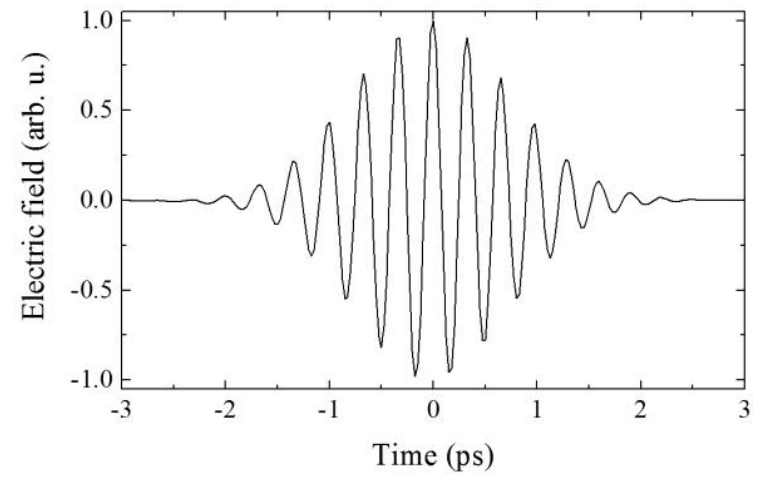

Fig. 6. Controlled multicycle waveform with $3 \mathrm{THz}$ carrier frequency, generated in $\mathrm{GaP}$ by two 1.5 -ps long $1.7-\mu \mathrm{m}$ wavelength pump pulses detuned in frequency by $3 \mathrm{THz}$.

(interaction length), but also demonstrate the versatility of GaP as a THz source. It is suitable to be used as a broadband source with high field strength, but also as a narrowband source with high spectral intensity. In the latter case, broadband tunability can also be provided by changing the PFT angle, possibly even up to as high as $7 \mathrm{THz}[36][37]$. The corresponding many-cycle waveform can be of advantage also in interesting new applications like charged-particle acceleration.

Multicycle $\mathrm{THz}$ pulses with controlled waveform can be generated for example by using two narrowband picosecond pump pulses detuned in frequency by the desired $\mathrm{THz}$ carrier frequency. One example, calculated with Model 1, is shown in Fig. 6. The controlled waveform with $3 \mathrm{THz}$ carrier frequency is generated in $\mathrm{GaP}$ by two 1.5-ps long $1.7-\mu \mathrm{m}$ wavelength pump pulses detuned in frequency by $3 \mathrm{THz}$, which also equals the phase-matching frequency. Suitable pump pulses can be generated for example as the signal and idler pulses of a nearlydegenerate optical parametric amplifier. Multicycle waveforms are needed for THz-driven electron acceleration in a waveguide [20].

\section{CONCLUSION}

Prompted by the recent demonstration of highly efficient intense semiconductor $\mathrm{THz}$ sources, here the most basic design aspects of such sources were addressed. Semiconductor THz sources pumped by infrared lasers or optical parametric amplifiers can reach similar efficiencies than LN-based sources, but can surpass this conventional technology in scalability to extremely high field strengths and energies, as well as in versatility and $\mathrm{THz}$ beam quality.

Optimization of pump pulse duration and pump intensity under the influence of 4PA was carried out in ZnTe and GaP. The dependence of the spectral features and electric field waveforms on the phase-matching frequency and crystal length were analyzed. Our results show that semiconductor $\mathrm{THz}$ sources can be flexibly tailored to applications, such as the generation of controlled multicycle waveforms for particle acceleration.

\section{REFERENCES}

[1] J. Hebling, G. Almási, I. Z. Kozma, and J. Kuhl, "Velocity matching by pulse front tilting for large-area THz-pulse generation," Opt. Lett., vol. 10, pp. 1161-1166, Oct. 2002.

[2] J. A. Fülöp, Z. Ollmann, C. Lombosi, C. Skrobol, S. Klingebiel, L. Pálfalvi, F. Krausz, S. Karsch, and J. Hebling, "Efficient generation of THz pulses with 0.4 mJ energy," Opt. Express, vol. 22, pp. 20155-20163, Aug. 2014.

[3] H. Hirori, A. Doi, F. Blanchard, and K. Tanaka, "Single-cycle terahertz pulses with amplitudes exceeding $1 \mathrm{MV} / \mathrm{cm}$ generated by optical rectification in $\mathrm{LiNbO}_{3}$," Appl. Phys. Lett., vol. 98, pp. 091106, Feb. 2011.

[4] M. Sajadi, M. Wolf, and T. Kampfrath, "Terahertz-field-induced optical birefringence in common window and substrate materials," Opt. Express, vol. 23, pp. 28985-28992, Nov. 2015.

[5] S.-W. Huang, E. Granados, W. R. Huang, K.-H. Hong, L. E. Zapata, and F. X. Kärtner, "High conversion efficiency, high energy terahertz pulses by optical rectification in cryogenically cooled lithium niobate," Opt. Lett., vol. 38, pp. 796-798, Mar. 2013.

[6] C. Vicario, A. V. Ovchinnikov, S. I. Ashitkov, M. B. Agranat, V. E. Fortov, and C. P. Hauri, "Generation of 0.9-mJ THz pulses in DSTMS pumped by a Cr: $\mathrm{Mg}_{2} \mathrm{SiO}_{4}$ laser," Opt. Lett., vol. 39, pp. 6632-6635, Dec. 2014.

[7] J. A. Fülöp, L. Pálfalvi, G. Almási, and J. Hebling, "Design of highenergy terahertz sources based on optical rectification," Opt. Express, vol. 18, pp. 12311-12327, Jun. 2010.

[8] M. I. Bakunov and S. B. Bodrov, "Terahertz generation with tilted-front laser pulses in a contact-grating scheme," J. Opt. Soc. Am. B, vol. 31, pp. 2549-2557, Nov. 2014.

[9] F. Blanchard, B. E. Schmidt, X. Ropagnol, N. Thiré, T. Ozaki, R. Morandotti, D. G. Cooke, and F. Légaré, "Terahertz pulse generation from bulk GaAs by a tilted-pulse-front excitation at $1.8 \mu \mathrm{m}$," Appl. Phys. Lett. vol. 105, pp. 241106, Dec. 2014.

[10] J. A. Fülöp, G. Polónyi, B. Monoszlai, G. Andriukaitis, T. Balciunas, A. Pugzlys, G. Arthur, A. Baltuska, and J. Hebling, "Highly efficient scalable monolithic semiconductor terahertz pulse source," Optica, vol. 3, pp. 1075-1078, Sep. 2016.

[11] Gy. Polónyi, B. Monoszlai, G. Gäumann, G. Andriukaitis, T. Balciunas, A. Pugzlys, A. Baltuska, T. Feurer, J. Hebling, and J. A. Fülöp, "Highenergy terahertz pulses from semiconductors pumped beyond the threephoton absorption edge," Opt. Express, vol. 24, pp. 23872-23882, Oct. 2016.

[12] F. Blanchard, L. Razzari, H.-C. Bandulet, G. Sharma, R. Morandotti, J.C. Kieffer, T. Ozaki, M. Reid, H. F. Tiedje, H. K. Haugen, and F. A. Hegmann, "Generation of $1.5 \mu \mathrm{J}$ single-cycle terahertz pulses by optical rectification from a large aperture ZnTe crystal," Opt. Express, vol. 15, pp. 13212-13220, Oct. 2007.

[13] Cs. Lombosi, Gy. Polónyi, M. Mechler, Z. Ollmann, J. Hebling, and J. A. Fülöp, "Nonlinear distortion of intense THz beams," New J. Phys., vol. 17, pp. 083041, Aug. 2015.

[14] K. Ravi, W. R. Huang, S. Carbajo, X. Wu, and F. X. Kärtner, "Limitations to THz generation by optical rectification using tilted pulse fronts," Opt. Express, vol. 22, pp. 20239-20251, Aug. 2014.

[15] L. Pálfalvi, J. A. Fülöp, G. Almási, and J. Hebling, "Novel setups for extremely high power single-cycle terahertz pulse generation by optical rectification," Appl. Phys. Lett. vol. 92, pp. 171107, Apr. 2008.

[16] Z. Ollmann, J. A. Fülöp, J. Hebling, and G. Almási, "Design of a highenergy terahertz pulse source based on ZnTe contact grating," Opt. Commun. vol. 315, pp. 159-163, Mar. 2014.

[17] P. Malevich, G. Andriukaitis, T. Flöry, A. J. Verhoef, A. Fernández, S. Alisauskas, A. Pugzlys, A. Baltuska, L. H. Tan, C. F. Chua, and P. B. Phua, "High energy and average power femtosecond laser for driving mid-infrared optical parametric amplifiers," Opt. Lett., vol. 38, pp. 27462749, Aug. 2013.

[18] M. Hemmer, D. Sánchez, M. Jelínek, V. Smirnov, H. Jelinkova, V. Kubecek, and J. Biegert, " $2-\mu \mathrm{m}$ wavelength, high-energy Ho:YLF chirped-pulse amplifier for mid-infrared OPCPA," Opt. Lett., vol. 40, pp. 451-454, Feb. 2015.

[19] L. Pálfalvi, J. A. Fülöp, G. Tóth, and J. Hebling, "Evanescent-wave proton postaccelerator driven by intense THz pulse," Phys. Rev. ST Accel. Beams, vol. 17, pp. 031301, Mar. 2014.

[20] E. A. Nanni, W. R. Huang, K.-H. Hong, K. Ravi, A. Fallhi, G. Moriena, R. J. D. Miller, and F. X. Kärtner, "Terahertz-driven linear electron acceleration," Nat. Commun., vol 6, pp. 8486, Oct. 2015. 
[21] T. Kampfrath, K. Tanaka, and K. A. Nelson, "Resonant and nonresonant control over matter and light by intense terahertz transients," Nat. Photonics, vol. 7, pp. 680-690, Aug. 2013.

[22] Q. Wu and X.-C. Zhang, "Ultrafast electro-optic field sensors," Appl. Phys. Lett., vol. 68, pp. 1604-1606, Mar. 1996.

[23] D. F. Nelson and E. H. Turner, "Electro-optic and piezoelectric coefficients and refractive index of Gallium Phosphide," J. Appl. Phys., vol. 39, pp. 3337-3343, Jun. 1968.

[24] D. T. F. Marple, "Refractive Index of ZnSe, ZnTe, and CdTe," J. Appl. Phys., vol. 35, pp. 539-541, Mar. 1964.

[25] E. D. Palik, Handbook of Optical Constants of Solids. New York: Academic Press, 1985, pp. 445-464.

[26] M. Schall, M. Walther, and P. Uhd Jepsen, "Fundamental and secondorder phonon processes in CdTe and ZnTe," Phys. Rev. B, vol. 64, pp. 094301, Sep. 2001.

[27] B. Bartal, I. Z. Kozma, A. G. Stepanov, G. Aalmáasi, J. Kuhl, E. Riedle, and J. Hebling, "Toward generation of $\mu \mathrm{J}$ range sub-ps THz pulses by optical rectification," Appl. Phys. B, vol. 86, pp. 419-423, Feb. 2007.

[28] K. L. Vodopyanov, "Terahertz-wave generation with periodically inverted gallium arsenide," Laser Phys., vol. 19, pp. 305-321, Feb. 2009.

[29] A. A. Said, M. Sheik-Bahae, D. J. Hagan, T. Wei, J. Wang, J. Young, and E. W. van Stryland, "Determination of bound-electronic and free-carrier nonlinearities in ZnSe, GaAs, CdTe, and ZnTe," J. Opt. Soc. Am. B, vol. 9, pp. 405-414, Mar. 1992.

[30] R. DeSalvo, A. A. Said, D. J. Hagan, E. W. van Stryland, and M. SheikBahae, "Infrared to ultraviolet measurements of two-photon absorption and $\mathrm{n}_{2}$ in wide bandgap solids," IEEE J. Quantum Electron., vol. 32, pp. 1324-1333, Aug. 1996.

[31] S. A. Ku, C. M. Tu, W.-C. Chu, C. W. Luo, K. H. Wu, A. Yabushita, C. C. Chi, and T. Kobayashi, "Saturation of free carrier absorption in ZnTe crystals," Opt. Express, vol. 21, pp. 13930-13937, Jun. 2013.

[32] M. C. Hoffmann, K.-L. Yeh, J. Hebling, and K. A. Nelson "Efficient terahertz generation by optical rectification at $1035 \mathrm{~nm}$," Opt. Express, vol. 15, pp. 11706-11713, Sep. 2007.

[33] J. A. Fülöp, L. Pálfalvi, M. C. Hoffmann, and J. Hebling, "Towards generation of mJ-level ultrashort $\mathrm{THz}$ pulses by optical rectification," Optics Express, vol. 19, pp. 15090-15097, Aug. 2011.

[34] C. Vicario, B. Monoszlai, Cs. Lombosi, A. Mareczko, A. Courjaud, J. A. Fülöp, and C. P. Hauri, "Pump pulse width and temperature effects in lithium niobate for efficient $\mathrm{THz}$ generation," Opt. Lett., vol. 38, pp. 5373-5376, 2013.

[35] R. L. Sutherland, Handbook of Nonlinear Optics. New York: Marcel Dekker, 2003.

[36] T. Tanabea, K. Suto, J. Nishizawa, K. Saito, and T. Kimura, "Tunable terahertz wave generation in the 3- to 7-THz region from GaP," Appl. Phys. Lett., vol. 83, pp. 237-239, Jul. 2003.

[37] J. Hebling, A. G. Stepanov, G. Almási, B. Bartal, and J. Kuhl, "Tunable $\mathrm{THz}$ pulse generation by optical rectification of ultrashort laser pulses with tilted pulse fronts," Appl. Phys. B, vol. 78, pp. 593-599, Mar. 2004.

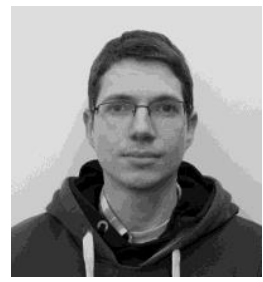

Gyula Polónyi received the M.S. degree in physics from the University of Pécs, Pécs, Hungary in 2012, where he is currently pursuing the Ph.D. degree in physics.

Since 2016 he has been a Research Assistant with the MTA-PTE High-Field Terahertz Research Group, Pécs, Hungary. His current research interest includes the development of intense pulsed semiconductor terahertz sources.

Mátyás I. Mechler received the M.S. degree in education of physics and mathematics in 2002 and the Ph.D. degree in physics in 2008 from the University of Pécs, Pécs, Hungary.

From 2002 to 2012, he was first a Research Assistant then a Research Fellow with the Institute of Physics, University of Pécs. Since 2012, he has been a Research Fellow with the
MTA-PTE High-Field Terahertz Research Group. He is the author of 17 articles and 2 inventions. His research interest includes ultrafast near-field optics, quantum entanglement, the theoretical study of periodic single-photon sources, and the modelling of terahertz sources.

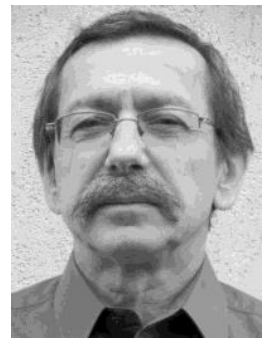

János Hebling (M) received the M.S. and $\mathrm{Ph} . \mathrm{D}$. degrees in physics from the József Attila (JATE) University, Szeged, Hungary, in 1978 and 1982, respectively.

He was with the JATE University. He was with the Max-Planck Institute for Solid State Research, Stuttgart, Germany, for more than six years and with the Massachusetts Institute of Technology, Cambridge for two years. He is with the University of Pécs, Pécs, Hungary, where he was the Head of the Experimental Physics Department from 1999 to 2010 and has been the Director of the Institute of Physics since 2008. Since 2012 he has been the Head of the MTA-PTE High-Field Terahertz Research Group. He is a Doctor of the Hungarian Academy of Sciences. He is the author of about 100 articles and 4 inventions. His research interests include ultrafast timeresolved spectroscopy of solids, nonlinear optics, generation and application of terahertz pulses, and the generation of waveform-controlled attosecond pulses.

Prof. Hebling is a Fellow of the Optical Society of America. He is a topical editor board of the Journal of the Optical Society of America B. He is the recipient of the Pál Selényi, the Széchenyi, and the Ányos Jedlik Awards.

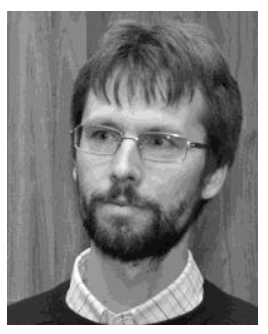

József A. Fülöp received the M.S. degree in physics and the Ph.D. degree in physics from University of Szeged, Szeged, Hungary, in 1998 and 2003, respectively.

He was a Research Assistant with the Max Planck Institute of Quantum Optics, Garching, Germany from 2001 to 2003 and with the Ludwig Maximilians University, Munich, Germany from 2003 to 2007. From 2007 to 2012 he was a Research Scientist with the University of Pécs, Pécs, Hungary. Since 2012 he has been a Senior Research Scientist with the MTA-PTE High-Field Terahertz Research Group, Pécs, Hungary. His research interest includes nonlinear optics, optical parametric amplification, the development of intense pulsed terahertz sources, and their applications.

Dr. Fülöp's awards and honors include a Fellowship of the German Academic Exchange Service (DAAD) and the János Bolyai Research Scholarship (Hungarian Academy of Sciences). 